\title{
2D/3D ultrasonography for endometrial evaluation in a cohort of 118 postmenopausal women with abnormal uterine bleedings
}

\author{
Grzegorz Stachowiak ${ }^{1}$, Agnieszka Zając ${ }^{1}$, Magdalena Pertynska-Marczewska ${ }^{2}$, \\ Tomasz Stetkiewicz ${ }^{1}$ \\ ${ }^{1}$ Department of Operative Gynecology and Gynecological Oncology, Polish Mother's Memorial Hospital Research Institute, Lodz, Poland \\ ${ }^{2}$ Independent Consultant in Obstetrics and Gynaecology, Private Practice, New Malden, United Kingdom
}

\begin{abstract}
Objectives: 2D/3D transvaginal ultrasonography in evaluation of endometrium in postmenopausal women with abnormal uterine bleedings (AUB).

Material and methods: 2D/3D transvaginal ultrasonography (TVU) was performed in 118 menopausal women with AUB. Endometrial volume and thickness, uterine volume and endometrial vascularity were evaluated. Complete histologic evaluation of the endometrium was obtained through dilatation \& curettage (D\&C) and/or hysteroscopy. Accordingly, patients were divided into 3 groups: controls (no endometrial pathology, $n=49$ ), Gl (benign endometrial pathology, $n=37$ ), GII (endometrial carcinoma, $\mathrm{n}=32$ ).

Results: Gll had greater thickness and volume of the endometrium, compared to Gl and controls. The presence of arterial vascular flow was identified only in GI and GII (51.35\% and 93.75\%, respectively). Endometrial volume merged together with uterine volume measurements (TVU-3D) showed a strong, statistical significance between GI and Gll, allowing differentiation of begin and malignant endometrial pathologies in postmenopausal women.

Conclusions: In TVU diagnostics of postmenopausal women with AUB the following play the most significant role: 1) endometrial thickness (TVU-2D); 2) endometrial volume (TVU-3D);3) uterine plus endometrial volume (TVU-3D); 4) vascularization within the endometrium, allowing to differentiate between pathological and normal endometrium (TVU-2/3D). Evaluation of the endometrial vascularity, both in TVU-2D and TVU-3D technique, does not allow for reliable differentiation between benign lesions and endometrial cancer.
\end{abstract}

Key words: transvaginal ultrasonography, endometrium, uterine bleeding, endometrial cancer, menopause

Ginekologia Polska 2016; 87, 12: 787-792

\section{INTRODUCTION}

Evaluation of abnormal uterine bleeding or postmenopausal bleeding with ultrasonography imaging has, in recent years, become a routine, non-invasive procedure and an initial evaluation in patients with abnormal uterine bleeding. This is especially enabled when transvaginal ultrasonography(TVU) became the standard examination in lieu of transabdominal ultrasonography.

This technique allows for endometrial receptivity assessment, which consists of description of endometrial pattern, endometrial thickness and/or endometrial volume [1].
TVU has also been used to qualitatively and quantitatively assess blood flow within the pelvic vessels and endometrium through the application of pulsed-wave Doppler (both 2-D [2] and 3-D power Doppler (3-DPD) maps) [3].

And although TVU does not meet the criteria for screening, along with the improvement in the examination techniques-introduction of more sophisticated equipment (including three-dimensional-3D devices) and skilled ultrasonographers - the diagnostic role of TUV in gynaecology is gaining an increasing importance and is not to be underestimated. 
This is particularly important in postmenopausal women, where not only benign pathology of the endometrium (polyps, endometrial growths) but also an increased incidence of neoplastic processes within the uterine cavity are observed. This relates primarily to Ca endometrii, the most common malignant neoplasm of the genital tract in women 50+ [4-6].

Ultrasound diagnostic of endometrial pathologies is particularly valuable not only in asymptomatic patients, where TVU evaluation would be the only indication for histopathological assessment (and possible diagnosis of the pathological process at an early stage of development), but also in women with symptoms of atypical spotting/bleeding from the genital tract. In such cases, TVU is not only a standard part of the initial evaluations in the management of abnormal uterine bleeding, but also an important component of the differential diagnosis, facilitating eligibility of patients to a particular type of approach: invasive, such as pipelle biopsy, dilatation \& curettage (D\&C), hysteroscopy or conservative.

\section{MATERIAL AND METHODS}

The study group consisted of 118 postmenopausal women (the absence of a period for one year), with abnormal uterine bleedings (metrorrhagia post menopausam) hospitalized in the Department of Gynecology and Gynecological Oncology, Polish Mother's Memorial Hospital — Research Institute (PMMH-RI), Lodz, Poland.

The study was carried out between 2012-2014 in the Department of Gynecology and Gynecologic Oncology as a statutory project number 2011/VI/5; and required an annual, positive opinion of the PMMH-RI Scientific Council (PMMH-RI Ethical Committee approval was not required). An annual report had been submitted to the Department of Science PMMH-RI, as well as to the Ministry of Health - where the study was registered (ID number 192897). Written informed consent was obtained from all patients. In all subjects a standard transvaginal ultrasound (TVU) in 2D (TVU-2D), combined with three-dimensional transvaginal evaluation (TVU-3D) of the endometrial and uterine volume was performed. TVU 2D/3D examination was performed using MEDISON ACCUVIX V10, with a three-dimensional 5-9-MHz transvaginal transducer. Endometrial thickness in TVU-2D was measured via longitudinal sections of the endometrial echo (double layer). Whereas, in TVU-3D evaluation a VOCAL technique (with $30^{\circ}$ scan rotation) was used.

In each case, the presence of vascularization within the endometrium (Power Doppler) was verified, and the specific indices for 2D and 3D evaluations were measured — including pulsatility index (vPI), resistance index (vRI), systole/diastole ratio (v S/D), vascularization index (VI), flow index (FI) and vascularization flow index (VFI). Power Dop-
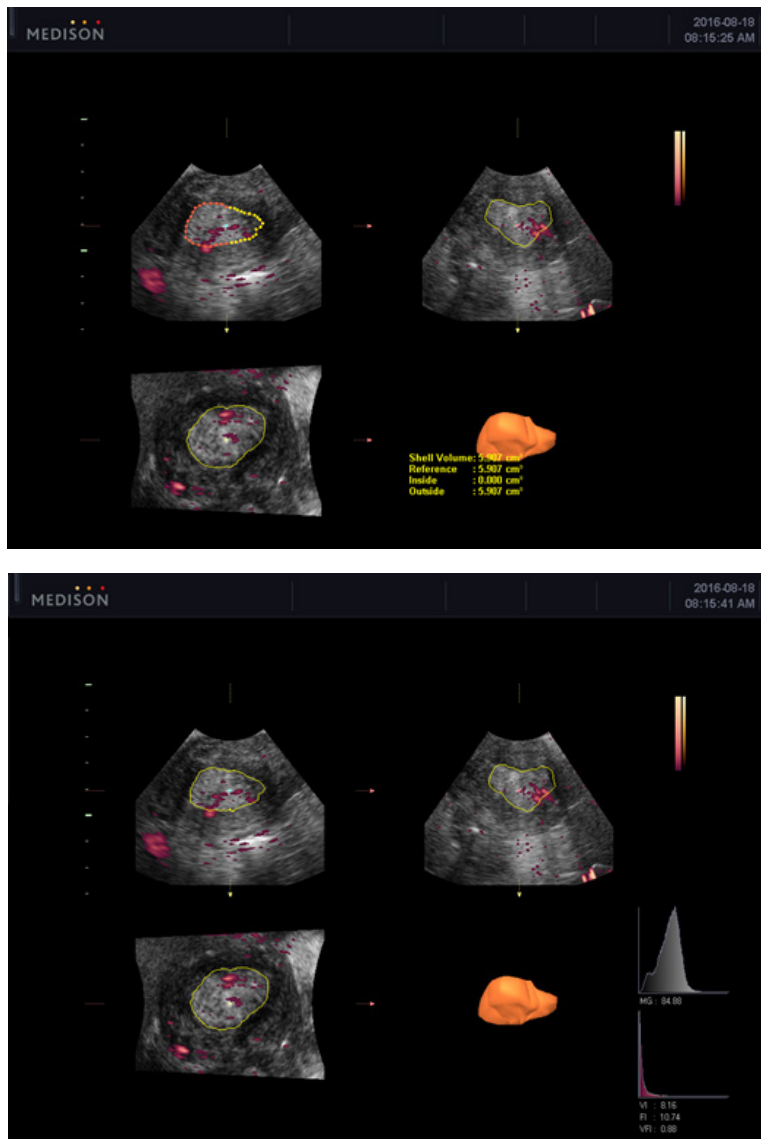

Figure 1. Examples of endometrial 3D imaging with VOCAL technique

pler settings were set to achieve maximum sensitivity for detecting low-velocity flow without noise (e.g. frequency, $5 \mathrm{MHz}$; power Doppler gain, 90; dynamic range, 70-90 dB; reject level, 2; colour map, type 2; frame average, 2; FSI, 1; density, low) (Fig. 1).

Patients with fibroids of a diameter $\geq 20 \mathrm{~mm}$ found in TVU-2D, resulting in an increase in uterus volume, had been disqualified from participating in the study.

Following the TVU, a standard hysteroscopy and/or D\&C was performed for complete histologic evaluation of the endometrium.

Hysteroscopy was performed by experienced hysteroscopists, using a $5 \mathrm{~mm}$ rigid hysteroscope and local anaesthesia was used if required.

Eventually, women eligible for the study, were further subdivided into three groups, based on the results of the histological examination: the control group (no endometrial pathology, $n=49$ ) and two study groups - Gl (benign endometrial pathology-endometrial polypus, endometrial hyperplasia, $n=37$ ) and $G$ II (endometrial carcinoma, $n=32$ ).

General characteristics of the study population, including classic Ca endometrii risk factors (such as obesity, diabetes, and arterial hypertension) are shown in Table 1. 
Table 1. General characteristic of the study groups (mean values \pm SD)

\begin{tabular}{|c|c|c|c|c|}
\hline Characteristics & $\begin{array}{c}\text { C - normal endometrium } \\
\text { (control group) }\end{array}$ & $\begin{array}{l}\text { G I - benign } \\
\text { endometrial pathology }\end{array}$ & $\begin{array}{l}\text { G II - endometrial } \\
\text { cancer }\end{array}$ & P-value \\
\hline Numbers & 49 & 37 & 32 & \\
\hline Mean age (years) & $60.41 \pm 10.86$ & $64.77 \pm 12.89$ & $66.41 \pm 7.95$ & $\begin{array}{c}P=0.270 \\
P^{*}=0.102 \\
P^{* *}=0.007\end{array}$ \\
\hline $\begin{array}{l}\text { LMP (menopause) } \\
\text { (years) }\end{array}$ & $49.99 \pm 3.61$ & $51.75 \pm 3.27$ & $51.01 \pm 4.24$ & $\begin{array}{c}P=0.152 \\
P^{*}=0.067 \\
P^{* *}=0.157\end{array}$ \\
\hline Menopausal age (years) & $13.48 \pm 12.56$ & $16.10 \pm 8.82$ & $15.39 \pm 9.41$ & $\begin{array}{c}P=0.311 \\
P^{*}=0.162 \\
P^{* *}=0.255\end{array}$ \\
\hline Arterial hypertension (\%) & $20(40.81 \%)$ & $16(43.24 \%)$ & $21(65.62 \%)$ & $\begin{array}{c}P=0.552 \\
P^{*}=0.632 \\
P^{* *}=0.425\end{array}$ \\
\hline Diabetes (\%) & $5(10.2 \%)$ & $4(10.81 \%)$ & $10(31.25 \%)$ & $\begin{array}{c}P=0.285 \\
P^{*}=0.219 \\
P^{* *}=0.112\end{array}$ \\
\hline Obesity (\%) & $13(26.53 \%)$ & $14(37.83 \%)$ & $18(55.25 \%)$ & $\begin{array}{c}P=0.635 \\
P^{*}=0.523 \\
P^{* *}=0.327\end{array}$ \\
\hline BMI $\left[\mathrm{kg} / \mathrm{m}^{2}\right]$ & $26.96 \pm 4.66$ & $29.42 \pm 6.05$ & $31.06 \pm 7.07$ & $\begin{array}{c}P=0.110 \\
P^{*}=0.068 \\
P^{* *}=0.004\end{array}$ \\
\hline
\end{tabular}

$\mathrm{P}^{*}$ - between G I and C; $\mathrm{P}^{* *}$ - between G II and C

The aim of this study was to assess the diagnostic efficiency of two-dimensional (2D) and three-dimensional (3D) transvaginal ultrasonography (TVS) in evaluating the endometrium of postmenopausal women with abnormal uterine bleedings.

\section{Statistics}

Statistical analysis was performed using Statistica 12.0 software package and $p<0.05$ was consider as indicative of significant difference. U Mann-Whitney's test and ANOVA Kruskal-Wallis' test were performed in order to evaluate the relationship between benign endometrial pathology or endometrial cancer and the examined parameters (patient characteristics: e.g. age, BMI, menopause, other diseases and obesity) in the studied patients. The results of studied parameters are presented as median \pm SEM and RQ means $\pm S D$ values. Statistica for Windows 10.0 program was applied for calculations considered as statistically significant.

\section{RESULTS}

The results obtained in the TVU-2D/3D ultrasound evaluations are shown in Table 2.

There was no statistically significant difference across the three groups of studied women for the parameter 'uterine volume'. However, for the parameter 'endometrial thickness' statistically significant differences across the three studied groups were observed, as indicated in detail in Figures 2 and 3.

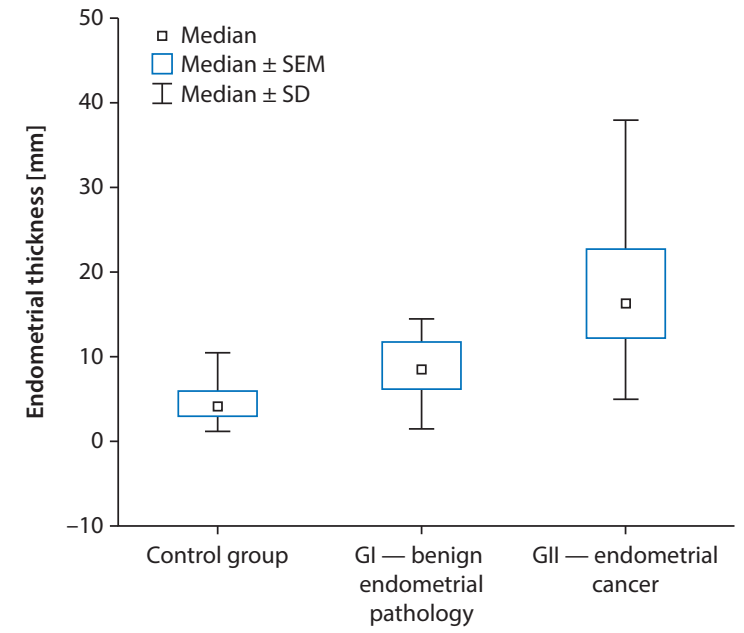

Figure 2. Endometrial thickness among the three groups

Moreover, for the parameter 'endometrial volume', there was also a statistically significant difference, especially when comparing groups Gll and C (Fig. 4).

When evaluating the two parameters'uterine volume'and 'endometrial volume' in TVU-3D together, a strong statistical significance between group GI (benign endometrium pathology) and Gll (Ca endometrii) p < 0.0001 was observed (Fig. 5).

Vascular flow indices in both techniques: TVU-2D (PI, $\mathrm{RI}, \mathrm{S} / \mathrm{D}$ ) and TVU-3D (VI, FI, VFI) showed no statistically significant differences between groups GI and G II. However, 
Table 2. Ultrasound parameters in the study groups (mean values \pm SD)

\begin{tabular}{|c|c|c|c|c|}
\hline USG-2D/USG-3D examination & $\begin{array}{l}\text { C- normal endometrium } \\
\text { (control group) }\end{array}$ & $\begin{array}{c}\text { G I - benign endometrial } \\
\text { pathology }\end{array}$ & G II - endometrial cancer & P-value \\
\hline Uterine volume $\left[\mathrm{cm}^{3}\right]$ & $37.45 \pm 36.18$ & $38.77 \pm 28.67$ & $69.02 \pm 45.86$ & $\begin{array}{c}P=0.407 \\
P^{*}=0.189 \\
P^{* *}=0.437\end{array}$ \\
\hline Endometrial thickness [mm] & $4.74 \pm 2.57$ & $9.5 \pm 5.45$ & $18.69 \pm 9.82$ & $\begin{aligned} P & =0.004 \\
P^{*} & =0.0002 \\
P^{* *} & =0.00005\end{aligned}$ \\
\hline Endometrial volume $\left[\mathrm{cm}^{3}\right]$ & $2.08 \pm 2.36$ & $4.37 \pm 4.23$ & $17.89 \pm 17.35$ & $\begin{array}{c}P=0.00061 \\
P^{*}=0.197 \\
P^{* *}=0.000007\end{array}$ \\
\hline Vascular flows (incl. arterial) & $2.04 \%(0 \%)$ & $54.05 \%(51.35 \%)$ & $100 \%(93.75 \%)$ & $\begin{array}{c}P=0.588 \\
P^{*}=0.453 \\
P^{* *}=0.745\end{array}$ \\
\hline RI & $(-)$ & $0.51 \pm 0.09$ & $0.43 \pm 0.08$ & $P^{* * *}=0.355$ \\
\hline PI & $(-)$ & $0.74 \pm 0.19$ & $0.64 \pm 0.17$ & $P^{* * *}=0.471$ \\
\hline S/D & $(-)$ & $2.10 \pm 0.24$ & $1.87 \pm 0.28$ & $P^{* * *}=0.471$ \\
\hline VI & $(-)$ & $0.94 \pm 1.36$ & $2.61 \pm 3.66$ & $P^{* * *}=0.098$ \\
\hline $\mathrm{FI}$ & $(-)$ & $26.89 \pm 3.45$ & $29.57 \pm 4.59$ & $P * * *=0.266$ \\
\hline VFI & $(-)$ & $0.18 \pm 0.37$ & $0.79 \pm 1.19$ & $P^{* * *}=0.082$ \\
\hline
\end{tabular}

$\mathrm{P}^{*}$ - between $\mathrm{GI}$ and $\mathrm{C} ; \mathrm{P}^{* *}$ — between G II a C; $\mathrm{P}^{* * *}$ — between $\mathrm{GI}$ and GII. In USG-3D technique uterine and endometrial volume were evaluated as well as VI, $\mathrm{FI}, \mathrm{VFI}$ indices; the remaining parameters were evaluated in USG-2D technique (endometrial thickness, blood flow indices — PI, RI, S/D)

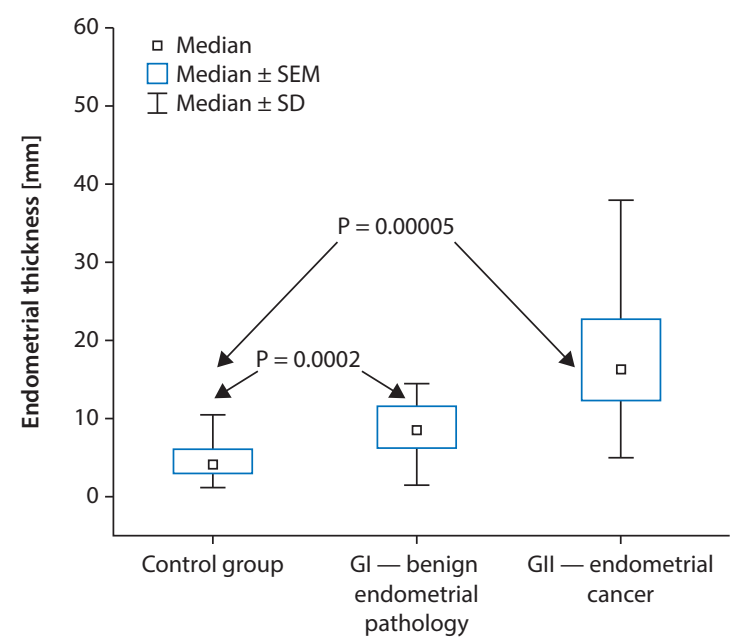

Figure 3. Endometrial thickness between the groups GI or GIl (benign endometrial pathology or Ca endometrii) and C (normal endometrium - control group)

it should be noted that the presence of arterial flow within the endometrium was related to endometrial pathology (benign or malignant).

\section{DISCUSSION}

Vaginal bleeding in postmenopausal women is not unequivocal with a diagnosis of endometrial cancer. Histopathological evaluation of the endometrium collected from women with AUB who participated in the current study showed that the largest group, representing $41.5 \%$ of

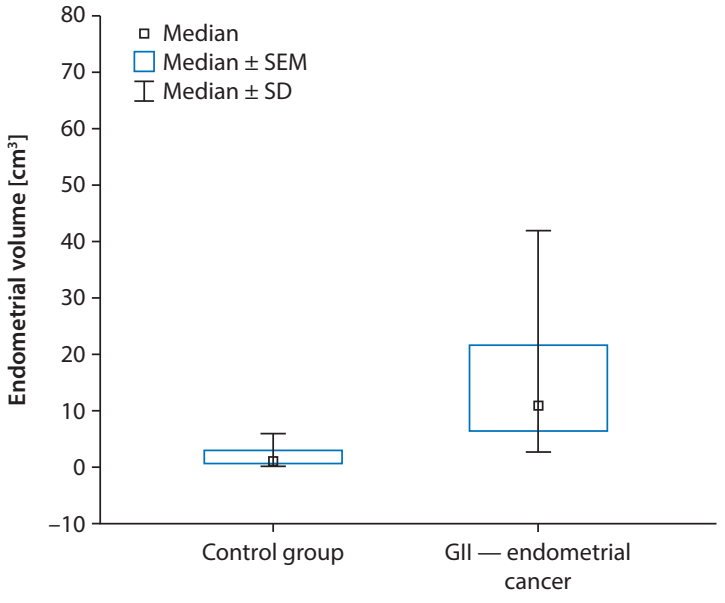

Figure 4. Endometrial volume between the two groups G II ( $\mathrm{Ca}$ endometrii) and C (normal endometrium - control group)

the study population, were women with normal endometrium, $31.4 \%$ of them were characterized by the presence of benign endometrial pathology (hyperplasia + polyps) and $27.1 \%$ were diagnosed with endometrial cancer. (However, the incidence of endometrial cancer in women with postmenopausal bleeding seems to be atypically high due to the oncological profile of the department).

There are certain risk factors for endometrial cancer. In this study, analysis of the general characteristics of the study population confirmed the well-known fact, that the C $a$ endometrii is associated with the so-called Virchow's triad; namely the presence of a higher incidence of arterial 


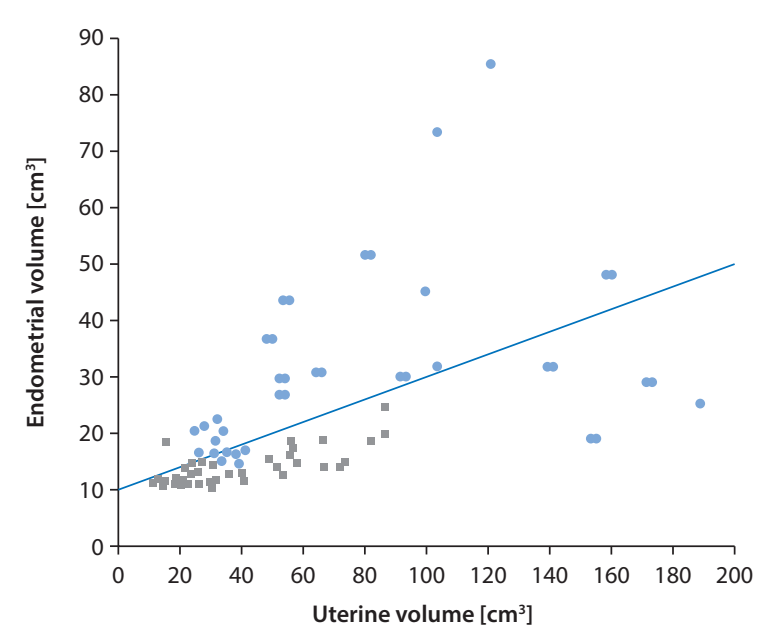

Figure 5. The uterine volume and endometrial volume in groups $\mathrm{GI}$ and G II (benign and Ca endometrii), green squares and orange dots accordingly

hypertension, diabetes and obesity in a group of patients with $\mathrm{Ca}$ endometrii. The incidence of these in the patient group G II was $65.62 \%, 31.25 \%$ and $55.25 \%$ respectively. (It seems that the lack of statistical significance between the studied groups with regards to the aforementioned parameters was due to the relatively small sample numbers). Importantly in our study, women with Ca endometrii (GII) were the only group with obesity, with a mean BMI of $31.06 \mathrm{~kg} / \mathrm{m}^{2}$ (a statistical significance between Gll and the control group was observed; $p=0.004)$. Such data are consistent with the latest reports [7]. Women in Gll group were also significantly older when compared to control group (an average of 6 years).

In postmenopausal women the normal endometrium is thin (4-5 mm thickness) and characterized by a small volume of approximately $2 \mathrm{~mL}$ [8]. In our study, the average thickness of normal endometrium was $4.74 \mathrm{~mm}$, with an average volume of $2.08 \mathrm{~mL}$, which is consistent with data in the literature [8].

Endometrial cancer is characterized by a significant increase in the thickness and volume of the endometrium [9], (here: over $13 \mathrm{~mL}$ ). Measurement of the endometrium by means of TVU-2D and TVU-3D techniques, is a reliable diagnostic tool in women with postmenopausal AUB. In this study, using TVU-2D technique we demonstrated statistically significant differences across all three groups of women. We showed that depending on the endometrial pathology, endometrial thickness increased accordingly, from $4.7 \mathrm{~mm}$ (on average) in the control group to $9.5 \mathrm{~mm}$ in Gl group (benign pathology) and to $18.69 \mathrm{~mm}$ in the GII (Ca endometrii). Using TVU-3D technique we demonstrated that the largest endometrial volume was observed in GII group (Ca endometrii), whereas the difference between GII-C groups reached a stronger statistical significance for TVU-3D (and endometrial volume) than for TVU-2D (and endometrial thickness). However, we did not notice a statistically significant difference between the control group and the $\mathrm{Gl}$ group (benign endometrial pathology).

Although the measurement of endometrial volume on its own was not able to distinguish between the studied groups GI and GII (lack of statistical significance, with higher volume in GII group), combining endometrial volume with uterus volume measurements appears to be an effective, complementary tool in the endometrial pathology evaluation.

Detection of vascular flow within the endometrium is a valuable component of the TVU 2D/3D examination. Available literature data from years 1991-2008 shows that the incidence of vascularization detected within the endometrium in patients with $\mathrm{Ca}$ endometrii varies within a wide range $40-90 \%$, and in the case of endometrial hyperplasia is $4-6 \%[8,10]$.

In the current study, endometrial vascularization was observed in $93.75 \%$ of women with endometrial cancer and in $51.35 \%$ of women with benign endometrial pathology (polyps + hyperplasia). The mere detection of arterial flow using power Doppler technique clearly demarcates the group of women with normal endometrium (here: lack of vascularization), from a group of women with benign or malignant endometrial pathology (here: the presence of vascularization).

In our study, evaluation of vascular flow characteristics in both techniques TVU-2D and TVU-3D was of less importance. Although the average values of flow indices (VI, FI, VFI) in TVU-3D technique were higher, and in TVU-2D technique ( $\mathrm{Pl}, \mathrm{Rl}, \mathrm{S} / \mathrm{D}$ ) lower in the Gll group (Ca endometrii) when compared with Gl group (benign endometrial pathology), the difference did not reach a statistical significance (the VFI parameter was the closest to reach statistical significance with $p=0.082$ ). Therefore, in our study, assessment of endometrium with pulsed-wave Doppler in 2D and 3D TVU did not enable distinction between benign and malignant endometrial pathology.

The results of this study are partly consistent with previous reports from Poland, where in women with endometrial hyperplasia or endometrial cancer, endometrium had been evaluated using TVU-2D/3D. This primarily concerns significantly higher values of the endometrium thickness and volume in the group with endometrial cancer when compared to the group with endometrial hyperplasia [11]. The researchers also found a significant correlation between the thickness of the endometrium and menopausal status, staging and grading of $\mathrm{Ca}$ endometrii (TVU-2D examination) [11]. This was not noticed for endometrial volume and the aforementioned parameters in TVU-3D technique [11]. Evaluation of blood flow indices within the endometrium in TVU-3D technique showed a statistically higher values for $\mathrm{VI}, \mathrm{FI}, \mathrm{VFI}$ in patients with endometrial cancer compared to 
patients with hyperplasia (the same correlation has been demonstrated for menopausal status and staging) [11]. For the blood flow indices in TVU-2D (PI, RI, PSV), evaluated in the uterine artery, there were no statistically significant differences for groups with cancer and hyperplasia nor for menopausal status, staging and grading of this cancer (exception: lower PSV in postmenopausal women) [11].

In another, somewhat earlier report, it was demonstrated that in TVU-3D mean values of vascular indices VI, Fl and VFI in the group of women with endometrial cancer were respectively 5.46, 25.99 and 1.89 and were significantly higher in comparison to the other studied groups of women (with normal endometrium, hyperplasia) [12]. The values of vascular indices $\mathrm{VI}, \mathrm{FI}$ and VFI in our study using TVU-3D technique were similar ( $\mathrm{VI}=2.61, \mathrm{FI}=29.57, \mathrm{VFI}=0.79$ ) and although they were higher than in the group with benign endometrial pathology, the values did not reach statistical significance.

In the current study we did not assess the depth of muscle tissue infiltration by a neoplastic process, however in the available literature on TVU-3D examination a tumour-free distance to serosa (TDS) index is used with the cut-off value of $9 \mathrm{~mm}$ [13].

In general, measurement of the endometrium by means of TVU-2D and TVU-3D techniques is a reliable diagnostic tool in women with postmenopausal AUB.

Although not very popular in gynaecology, three-dimensional transvaginal examination (TVU-3D), when used to evaluate in particular endometrial volume and/or combining the above parameter with uterine volume, seems to increase the accuracy of endometrium pathology diagnosis at the early stage. This, in turn, should have an impact on the choice of a particular therapeutic measure.

\section{REFERENCES}

1. Polanski LT, Baumgarten MN, Brosens JJ, [et al.]. Assessment of Endometrial Vascularity Using Spatiotemporal Image Correlation: A Study Comparing Spherical Sampling and Whole-Tissue Analysis. Ultrasound Med Biol. 2015, 41, 2798-2805.

2. Applebaum M.The uterine biophysical profile. Ultrasound Obstet Gynecol. 1995, 5, 67-68.

3. Ledee N, Chaouat G, Serazin V, [et al.]. Endometrial vascularity by three-dimensional power Doppler ultrasound and cytokines: A complementary approach to assess uterine receptivity. J Reprod Immunol. 2008, 77, 57-62.

4. Sheikh MA, Althouse AD, Freese KE, [et al.]. USA endometrial cancer projections to 2030: should we be concerned? Future Oncol. 2014, 10, 2561-2568.

5. Crosbie E, Morrison J.The emerging epidemic of endometrial cancer:Time to take action. Cochrane Database Syst Rev. 2014 Dec 22, 12, ED000095.

6. AlHilli MM, Bakkum-Gamez JN, Mariani A, [et al.]. Risk-adjusted outcomes in elderly endometrial cancer patients: implications of the contrasting impact of age on progression-free and cause-specific survival. Gynecol Oncol. 2015, 138, 133-140.

7. Jenabi $E$, Poorolajal J. The effect of body mass index on endometrial cancer: a meta-analysis. Public Health. 2015, 129, 872-880.

8. Benacerraf BR, Shipp TD, Bromley B. Which patients benefit from a 3D reconstructed coronal view of the uterus added to standard routine $2 \mathrm{D}$ pelvic sonography? AJR Am J Roentgenol. 2008, 190, 626-629.

9. Brązert J, Pietryga M. Obrazowanie endometrium. In: Podstawy praktycznej ultrasonografii w ginekologii i położnictwie. Wydawnictwo Exemplum. 2009, 85-90.

10. Grunfeld L, Walker B, Bergh PA, [et al.]. High-resolution endovaginal endometrium: A noninvasive test for endometrial adequacy. Obstet Gynaecol. 1991, 78, 200-204.

11. Chróściel M, Czekierdowski A. Badanie ultrasonograficzne sondą przezpochwową i sonografia 3D u kobiet ze zmianami rozrostowymi endometrium. Przegl Menopauzalny. 2008, 4, 210-216.

12. Stachowicz N, Czekierdowski A, Daniłoś J, [et al.]. Three-dimensional sonoangiography in diagnostic of endometrial hyperplasia and carcinoma. An assessment of vascularization indices and endometrial volume. Przegl Lek. 2005, 62, 827-829.

13. Alcazar JL, Pineda $\mathrm{L}$, Martinez-Astorquiza Corral T, [et al.]. Transvaginal/transrectal ultrasound for assessing myometrial invasion in endometrial cancer: a comparison of six different approaches. J Gynecol Oncol. 2015, 26, 201-207. 